\title{
Evaluation of corrosion inhibition efficiency of some novel Schiff bases through a proposed QSAR model: DFT investigations supported by weight loss technique
}

\author{
M.H. Hadizadeh ${ }^{1,2 *}$ and M. Hamadanian ${ }^{3,4}$ \\ ${ }^{1}$ Hefei National Laboratory of Physical Sciences at the Microscale, School of Chemistry \\ and Materials Sciences, University of Science and Technology of China, Hefei, Anhui \\ 230026, China \\ ${ }^{2}$ Hefei National Laboratory for Physical Sciences at the Microscale, Synergetic Innovation \\ Center of Quantum Information \& Quantum Physics, University of Science and \\ Technology of China, Hefei, Anhui 230026, China \\ ${ }^{3}$ Institute of Nanoscience and Nanotechnology, University of Kashan, Kashan, Islamic \\ Republic of Iran \\ ${ }^{4}$ Department of Physical Chemistry, Faculty of Chemistry, University of Kashan, Kashan, \\ Islamic Republic of Iran \\ *E-mail: hadimh@mail.ustc.edu.cn
}

\begin{abstract}
Organic molecules having some functional groups, double and triple bonds in their structures have high capabilities in preventing and inhibiting metal corrosion in various corrosive solutions. The conventional Quantitative Structure Activity Relationship (QSAR), is known as a worthwhile model to foretell corrosion inhibition efficiency. In some cases, the results obtained from the conventional QSAR model are significantly different from laboratory values. The corrosion inhibition efficiency of a novel Schiff base namely (E)-N1-(thiophen-2ylmethylene)-N3,N3-bis(2-((E)-(thiophen-2-ylmethylene)amino)ethyl)propane-1,3-diamine with its derivatives were studied using density functional theory (DFT) calculations and QSAR approach. The quantum chemical parameters including the energies of the molecular orbital, the highest occupied molecular orbital, the lowest unoccupied molecular orbital and dipole moment were calculated to investigate the inhibition efficiency of inhibitors. The results showed that this compound and its derivatives have high corrosion inhibition efficiency especially at high concentrations. Noticeable correlation was obtained between corrosion inhibition efficiency and quantum chemical parameters through B3LYP/6-31G** method. The obtained theoretical results were in good agreement with the experimental findings. Moreover, we proposed a modified QSAR (MQSAR) model and evaluated it by our novel Schiff bases as well as some derivatives of triazole, oxadiazole and thiadiazole. The results obtained through MQSAR were in a better agreement with experimental inhibition efficiency values.
\end{abstract}

Keywords: Schiff bases, QSAR, corrosion, DFT, HOMO.

Received: August 24, 2021. Published: October 28, 2021

doi: $\underline{10.17675 / 2305-6894-2021-10-4-9}$ 


\section{Introduction}

Mild steel, also sometimes called low-carbon steel, is known as the most common form of steel used in the industry due to its relatively low price [1]. Acidic solutions specially sulfuric and hydrochloric acids are extensively used in industrial chemical processes such as acid cleaning, descaling, pickling and drilling operations in oil and gas exploration [2]. The challenge is that mild steel has low corrosion resistance specifically in acidic environments. During the last five decades, the corrosion of mild steel and the method of preventing its corrosion in acid solutions has been widely researched as an important issue [3-12]. Some organic compounds are known as effective inhibitors for corrosion inhibition of mild steel because of the existence of $\pi$-electrons and also functional groups in their structures $[13,14]$. Schiff bases are a bunch of organic compounds that due to the presence of the $\mathrm{C}=\mathrm{N}$ group and also electronegative atoms in their structures are becoming more desirable as efficient inhibitors for corrosion of mild steel in hydrochloric acid medium [15, 16]. Computational methods are frequently used to investigate structural and inhibiting properties of desired structures/systems. Density functional theory (DFT) is an influential tool for designing, prediction and analysis of organic corrosion inhibitors as well as nanostructures [17-22]. Linear and non-linear QSAR models using quantum chemical parameters have been used during the past several years in order to evaluate the inhibition efficiency of some Schiff bases [23-25]. Unfortunately, in some cases, there is still a significant gap between experimental and theoretical values obtained from conventional QSAR models. Accordingly, following our previous experimental and theoretical studies [26-29], DFT using hybrid B3LYP/6-31G** as a higher level of theory were carried on a novel Schiff base (E)-N1-(thiophen-2-ylmethylene)-N3,N3-bis(2-((E)-(thiophen-2-ylmethylene)amino)ethyl) propane-1,3-diamine along with its derivatives, as corrosion inhibitors for application in $\mathrm{HCl}$ medium. All structures were synthesized through a well-known procedure [30]. Where, the experimental inhibition efficiency $\left(I E_{\exp } \%\right)$ was measured at different concentrations, including $5,10,50,100,500$ and $1000 \times 10^{-7} \mathrm{M}$. Indeed, the major aim of this paper is to minimize the significant gap between experimental and theoretical values obtained from a quantitative structural activity relationship by one proposed model.

\section{Experimental Details}

In order to determine inhibition efficiency, weight loss measurements were used in a doublewalled glass cell furnished with a thermostat cooling condenser at different immersion times. Where, the maximum immersion time was about one day at a temperature of $25^{\circ} \mathrm{C}$ while immersion time at the other temperatures was about one hour. Specimens containing different concentrations of the inhibitors were immersed in $50 \mathrm{ml} 2 \mathrm{M} \mathrm{HCl}$ solution. The mass of the specimens was evaluated before and after immersion through an analytical balance accurate to $0.01 \mathrm{mg}$. The corrosion rate $(v)$ was calculated through the following equation [30]: 


$$
v=\frac{m_{1}-m_{2}}{S \cdot t}
$$

where $m_{1}$ and $m_{2}$ are the mass of the specimen before and after corrosion, respectively, $S$ is the total area of the specimen and $t$ is corrosion time.

The corrosion inhibition efficiency of inhibitors was obtained by substituting the corrosion rate $(v)$ in the following equation [30]:

$$
I E_{\exp } \%=\frac{v_{0}-v}{v} \cdot 100
$$

where $v_{0}$ and $v$ are the corrosion rates of mild steel samples in $2 \mathrm{M}$ hydrochloric acid without and with the Schiff bases, respectively.

\section{Computational Details}

Quantum chemical calculations have already confirmed to be very beneficial for the evaluation, molecular structure and electrostatic interaction of a molecule with a solvent. The quantum chemical calculations were carried out in Gaussian 09 program [31], using density functional theory (DFT) with the Beck's parameter hybrid functional $(B)$ and the Lee-Yang-Parr correlation functional $(L Y P)$ called as $B L Y P$ functional [32,33], with 6-31G** basis set. After a complete geometry optimization on our novel Schiff base and its derivatives Figure 1, the quantum chemical parameters including the energies of the molecular orbital, the highest occupied molecular orbital $\left(E_{\mathrm{HOMO}}\right)$, the lowest unoccupied molecular orbital $\left(E_{\mathrm{LUMO}}\right)$ and $\mu$ (dipole moment) were calculated to investigate the inhibition efficiency of inhibitors. The highest occupied molecular orbital is often associated with the electron-donating ability of a structure while the lowest unoccupied molecular orbital indicates the ability to accept electrons. Therefore, increasing the value of $E_{\mathrm{HOMO}}$ causes the electron donating tendency of the Schiff base to the orbital of the mild steel atoms. Reciprocally, the low values of $E_{\mathrm{LUMO}}$ specify the capacity to accept electrons from the orbital of the mild steel atoms. The objective of the present study is to investigate the inhibitory effects of (E)-N1-(thiophen-2-ylmethylene)-N3,N3-bis(2-((E)-(thiophen-2ylmethylene)amino)ethyl)propane-1,3-diamine, shown by $\mathrm{SB}$, along with some of its derivatives (SB-R1, SB-R2, SB-R3), Figure 1. In order to determine theoretical inhibition efficiency $\left(I E_{\text {the }} \%\right)$ based on the conventional non-linear QSAR model, the solvent effects were applied by optimizing the $\mathrm{B} 3 \mathrm{LYP} / 6-31 \mathrm{G} * *$ gas-phase stationary points using the most popular self-consistent reaction field (SCRF) calculations [34-36] based on the polarizable continuum model of Tomasi's group [37, 38]. 


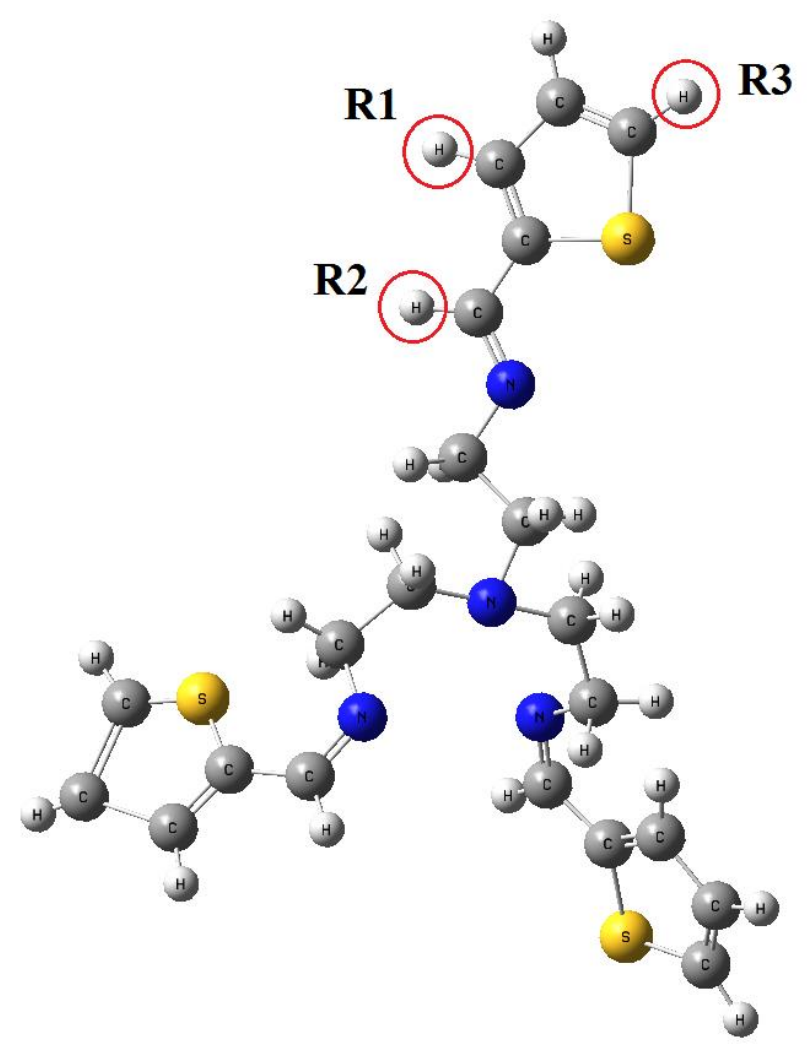

Figure 1. Optimized geometry of $(E)$ - $N 1$-(thiophen-2-ylmethylene)- $N 3, N 3$-bis(2-( $(E)$ (thiophen-2-ylmethylene)amino)ethyl)propane-1,3-diamine. Where, R1, R2 and R3 indicate the location of $\mathrm{CH}_{3}$ and $\mathrm{NO}_{2}$ functional groups on one branch as well as the other two branches of structure.

\section{Results and Discussion}

The corrosion inhibition efficiencies for SB-R1, SB-R2 and SB-R3 were determined using weight loss measurements. The experimental values $\left(I E_{\exp } \%\right)$ showed that as the inhibitor concentration increases, the corrosion inhibition efficiencies also increase dramatically. So that the maximum efficiency for structures SB-R1, SB-R2 and SB-R3 occurred at a concentration of $1000 \cdot 10^{-7} \mathrm{M}$, equal to $91.41 \%, 88.80 \%$ and $93.21 \%$, respectively, Table 1 . After performing high level optimization, the quantum chemical parameters of our new Schiff base (SB) along with its derivatives were calculated, Table 2. Based on the results, the most structural energy is related to the structure of SB-R3. One of the reasons for this high stability could be the steric repulsion of $\mathrm{NO}_{2}$ group. A simple measure of the accuracy of the electron density of a polar molecule can be determined by dipole moment. SB-R3 also has a higher dipole moment than other structures, which indicates its impressive effect on the interactions with other molecules. Moreover, the values of $E_{\mathrm{HOMO}}$ show that the electron donating tendency of SB-R3 to the orbital of the mild steel atoms is more than other Schiff bases. In all structures (SB-R1, SB-R2 and SB-R3) the electron donating sites are located in the central core, where the HOMO orbitals are concentrated, Figure 2. 

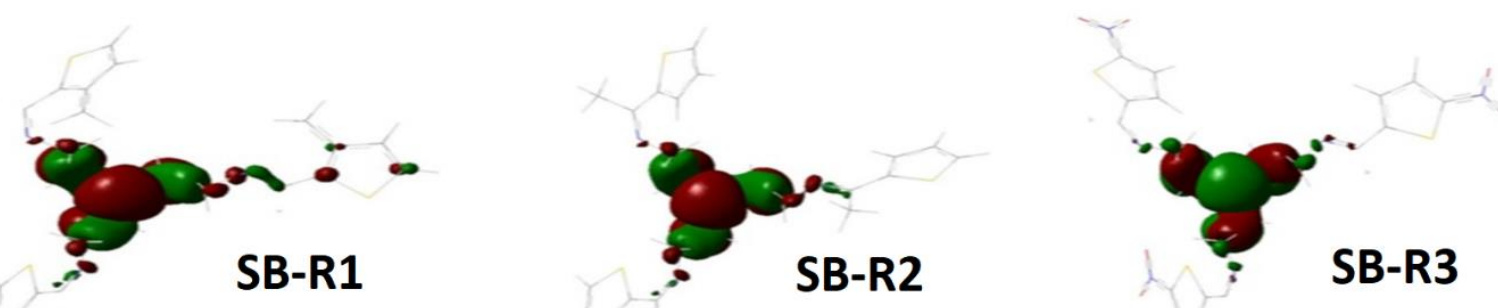

Figure 2. HOMO visualization of Schiff bases SB-R1, SB-R2 and SB-R3. Where, R1 and R2 replaced with the $\mathrm{CH}_{3}$ functional group, $\mathrm{R} 3$ Replaced with $\mathrm{NO}_{2}$ functional group on one branch as well as the other two branches of structure.

Table 1. The theoretical inhibition efficiency based on conventional $\left(I E_{\text {the }} \%\right)$ and modified ( $\left.I E_{\text {the }}^{*} \%\right)$ QSAR models, where $E_{\mathrm{r}}$ and $E_{\mathrm{r}} *$ are relative errors related to conventional and modified QSAR models, respectively.

\begin{tabular}{|c|c|c|c|c|c|c|}
\hline Inhibitor & $C(\mathbf{M}) \times 10^{7}$ & $I E_{\exp } \%$ & $I E_{\text {the }} \%$ & $I E_{\text {the }}^{*} \%$ & $E_{\mathbf{r}}$ & $E_{\mathrm{r}}^{*}$ \\
\hline \multirow{6}{*}{ SB-R1 } & 5 & 61.29 & 52.20 & 57.72 & 14.83 & 5.825 \\
\hline & 10 & 64.30 & 68.59 & 65.87 & 6.672 & 2.442 \\
\hline & 50 & 79.89 & 91.61 & 81.19 & 14.67 & 1.627 \\
\hline & 100 & 86.13 & 95.62 & 85.92 & 11.02 & 0.244 \\
\hline & 500 & 90.45 & 99.09 & 93.17 & 9.552 & 3.007 \\
\hline & 1000 & 91.41 & 99.54 & 95.07 & 8.894 & 4.004 \\
\hline \multirow{6}{*}{ SB-R2 } & 5 & 66.15 & 58.40 & 62.19 & 11.72 & 5.986 \\
\hline & 10 & 70.25 & 73.74 & 69.93 & 4.953 & 0.455 \\
\hline & 50 & 83.29 & 93.35 & 83.87 & 12.08 & 0.696 \\
\hline & 100 & 85.73 & 96.56 & 88.03 & 12.63 & 2.683 \\
\hline & 500 & 86.96 & 99.29 & 94.27 & 14.18 & 8.406 \\
\hline & 1000 & 88.80 & 99.64 & 95.88 & 12.21 & 7.973 \\
\hline \multirow{6}{*}{ SB-R3 } & 5 & 46.00 & 37.49 & 47.06 & 18.50 & 2.304 \\
\hline & 10 & 58.71 & 54.53 & 55.69 & 7.120 & 5.144 \\
\hline & 50 & 67.25 & 85.71 & 73.76 & 27.45 & 9.680 \\
\hline & 100 & 83.73 & 92.30 & 79.90 & 10.23 & 4.574 \\
\hline & 500 & 91.27 & 98.36 & 89.88 & 7.768 & 1.523 \\
\hline & 1000 & 93.21 & 99.17 & 92.63 & 6.394 & 0.622 \\
\hline
\end{tabular}


Table 2. The total energy, dipole moment, HOMO and LUMO energy using B3LYP/6-31G** related to optimized structures in the solution phase.

\begin{tabular}{cccccc}
\hline Inhibitor & Group & $\boldsymbol{E}($ Hartree $)$ & $\boldsymbol{\mu}(\mathbf{D})$ & $\boldsymbol{E}_{\text {HOMо }}(\mathbf{e v})$ & $\boldsymbol{E}_{\text {LUMo }}(\mathbf{e v})$ \\
\hline $\mathrm{SB}$ & $\mathrm{H}$ & -2228.5 & 5.5141 & -0.21576 & -0.06284 \\
$\mathrm{SB}-\mathrm{R}_{1}$ & $\mathrm{CH}_{3}$ & -2346.5 & 3.7707 & -0.21660 & -0.06194 \\
$\mathrm{SB}-\mathrm{R} 2$ & $\mathrm{CH}_{3}$ & -2346.5 & 4.4270 & -0.21128 & -0.05372 \\
$\mathrm{SB}-\mathrm{R} 3$ & $\mathrm{NO}_{2}$ & -2842.1 & 11.1899 & -0.23749 & -0.13260 \\
\hline
\end{tabular}

In the next step, many attempts were made to find a relationship between quantum chemical parameters and experimental corrosion inhibition efficiencies through multiple linear regressions for the different models. Investigations have shown that there is no direct linear relationship between inhibition efficiency and the calculated quantum chemical parameters. This indicates that the inhibition efficiencies of the inhibitors depend on composite functions that cannot be adequately expressed by linear models. The conventional linear equation for calculating corrosion inhibition efficiency $\left(E_{\mathrm{cal}} \%\right)$ based on quantum chemical parameters is as follows [39]:

$$
E_{\mathrm{cal}}=A x_{j} C_{i}+B
$$

Where, $A$ and $B$ are constant parameters that will be calculated by regression analysis; $x_{\mathrm{j}}$ is a quantum chemical index characteristic of molecule $j$; and $C_{\mathrm{i}}$ defined as the concentration of the inhibitor. Although this linear model was found to be insufficient for deriving relation between the results obtained in this study, the conventional nonlinear model proposed by Lukovits et al. was found to be satisfactory to investigate corrosion inhibitors. Indeed, this model predicts the inhibition efficiency of inhibitors through making nonlinear relation between some quantum chemical parameters such as $E_{\mathrm{HOMO}}, E_{\mathrm{LUMO}}$ and dipole moment of inhibitors in different concentration as follows [40]:

$$
I E_{\mathrm{the}} \%=\frac{\left(A E_{\mathrm{HOMO}}+B E_{\mathrm{LUMO}}+C \mu+D\right) C_{\mathrm{inh}}}{\left[1+\left(A E_{\mathrm{HOMO}}+B E_{\mathrm{LUMO}}+C \mu+D\right) C_{\mathrm{inh}}\right]}
$$

Where $I E_{\text {the }} \%, E_{\text {HOMO }}, E_{\mathrm{Lumo}}, \mu$ and $C_{\text {inh }}$ are inhibition efficiency, HOMO energy, LUMO energy, dipole moment and concentration of inhibitor, respectively. The constant parameters of $A, B, C, D$ are related to regression coefficients that determine through fitting analysis. The coefficients obtained from the Equation 4 through fitting regression parameters were equal to $A=18372171.7, B=40043518.5, C=300413.968$ and $D=7510841.01$. Substituting these values into the principal relation gives the following equation:

$$
I E_{\mathrm{the}} \%=\frac{\left(18372171.7 E_{\mathrm{HOMO}}+40043518.5 E_{\mathrm{LUMO}}+300413.968 \mu+7510841.01\right) C_{\mathrm{inh}}}{\left[1+\left(18372171.7 E_{\mathrm{HOMO}}+40043518.5 E_{\mathrm{LUMO}}+300413.968 \mu+7510841.01\right) C_{\mathrm{inh}}\right]}
$$


The results obtained from theoretical inhibition efficiency $\left(I E_{\text {the }} \%\right)$ related to three Schiff bases SB-R1, SB-R2 and SB-R3 in different concentrations are summarized in Table 1. With a simple review it can be seen that the relative error $\left(E_{\mathrm{r}}\right)$, for these three Shiff bases at a concentration of $5 \cdot 10^{-7} \mathrm{M}$ has the lowest value while in other concentrations this relative error $\left(E_{\mathrm{r}}\right)$ is significant. Thus, although the inhibition efficiency based on conventional nonlinear model can be predicted, there is still a significant relative error between the theoretical and experimental values.

In order to minimize relative errors, various analytical methods using different software were applied to several Schiff bases in different concentrations. The results showed that this gap (Difference between the theoretical and experimental inhibition efficiencies) can be significantly reduced after applying the second root of the concentration values of the conventional non-linear QSAR model, called as modified QSAR (MQSAR), Equation 6.

$$
I E_{\mathrm{the}}^{*} \%=\frac{\left(A E_{\mathrm{HOMO}}+B E_{\mathrm{LUMO}}+C \mu+D\right) \sqrt{C_{\mathrm{inh}}}}{\left[1+\left(A E_{\mathrm{HOMO}}+B E_{\mathrm{LUMO}}+C \mu+D\right) \sqrt{C_{\mathrm{inh}}}\right]}
$$

Where, $I E_{\text {the }}^{*}$ is defined as modified inhibition efficiency. $E_{\text {номо, }}, E_{\mathrm{LUMO}}, \mu$ and $C_{\mathrm{inh}}$ are HOMO energy, LUMO energy, dipole moment and concentration of inhibitor, respectively. Where $A, B, C, D$ are constant parameters related to regression coefficients. The fitting analysis using Equation 6 for SB-R1, SB-R2 and SB-R3 in different concentrations led to the coefficient values of $A=11672.1427, B=25667.6689, \quad C=186.562062$ and $D=5345.05300$. So that after placing these values, the following equation is obtained:

$$
I E_{\text {the }}^{*} \%=\frac{\left(11672.1427 E_{\text {НОмО }}+25667.6689 E_{\mathrm{LUMO}}+186.562062 \mu+5345.05300\right) \sqrt{C_{\text {inh }}}}{\left[1+\left(11672.1427 E_{\text {НОМО }}+25667.6689 E_{\mathrm{LUMO}}+186.562062 \mu+5345.05300\right) \sqrt{C_{\text {inh }}}\right]}(7)
$$

The results obtained from the modified inhibition efficiency ( $\left.I E_{\text {the }}^{*} \%\right)$ related to SB-R1, SB-R2 and SB-R3 in different concentrations are summarized in Table 1. It is clear that at different concentrations of all Schiff bases, the relative errors obtained from MQSAR are much less than relative errors obtained from QSAR model. In order to make a statistical comparison between the relative errors obtained from QSAR and MQSAR models, their conical diagrams are depicted in Figure 3. Where, the relative errors with respect to MQSAR and QSAR models are identified by red and blue cones, respectively.

In order to confirm the above results, in addition to our structures, these two conventional and modified models (QSAR and MQSAR) were also evaluated by some structures such as triazole and oxadiazole and thiadiazole derivatives (Figure 4). The experimental values of corrosion inhibition related to these twelve Schiff bases were collected from different literatures [41-50]. 


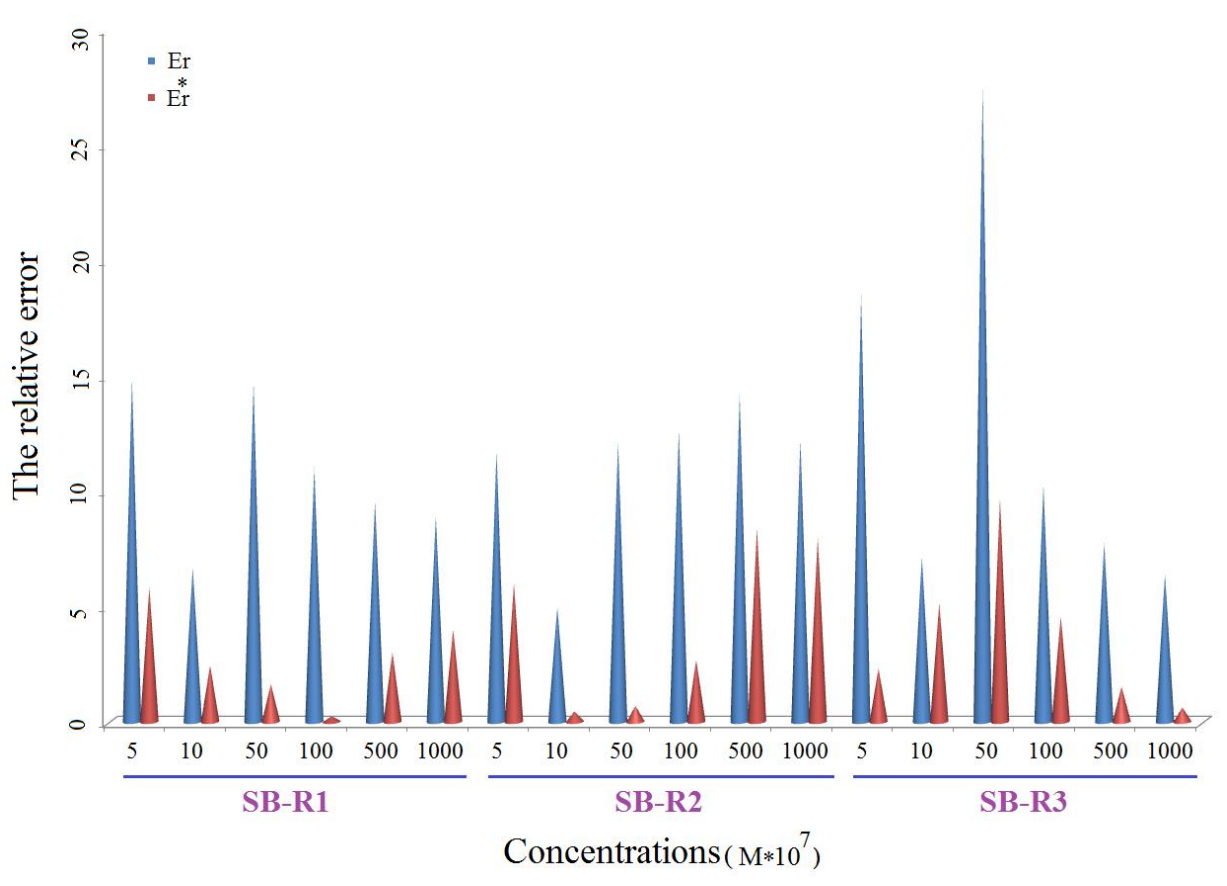

Figure 3. A comparison between relative errors for three Schiff bases SB-R1, SB-R2 and SBR3 in Different concentrations. Where, the blue and red cones are related to QSAR and MQSAR models, respectively.
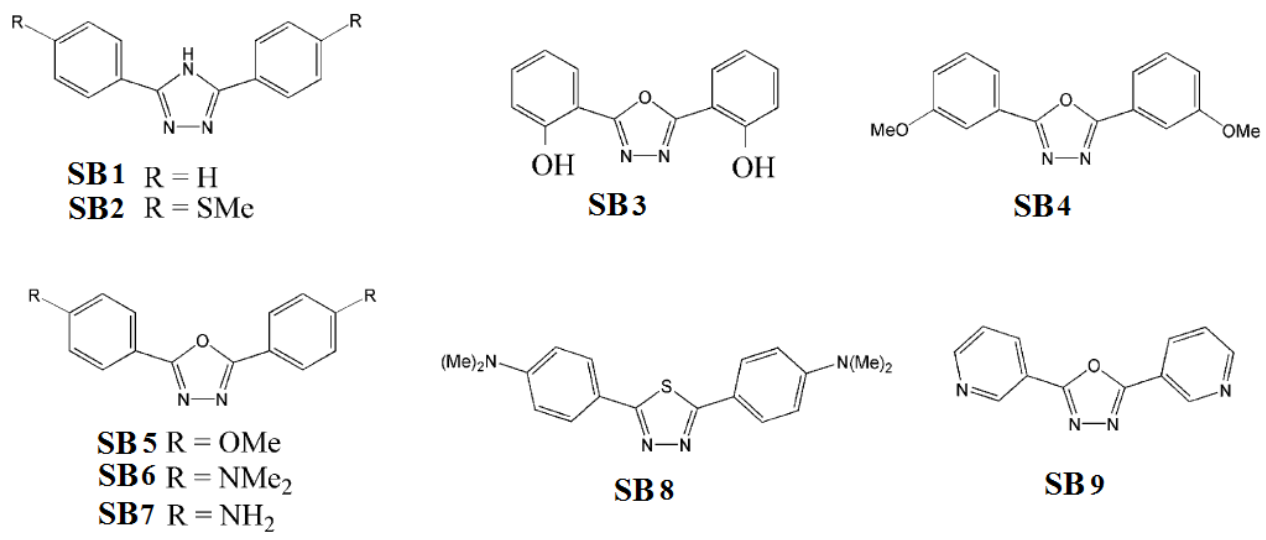

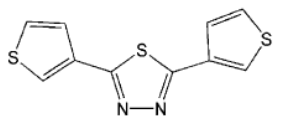

SB 10

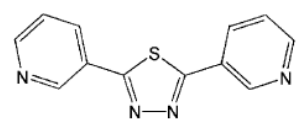

SB 11

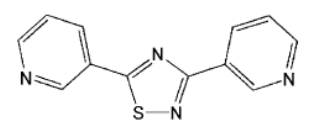

SB12

Figure 4. The structures of the triazole, oxadiazole and thiadiazole derivatives having experimental corrosion inhibition efficiencies.

The quantum chemical parameters of these twelve Shiff bases after a full optimization using the $\mathrm{B} 3 \mathrm{LYP} / 6-31 \mathrm{G}^{* *}$ are given in Table 3. The HOMO energy values show an almost identical and high electron donating tendency of Schiff bases SB9, SB11 and SB12. Meanwhile, SB2 has a higher dipole moment than other structures, which indicates its impressive effect on the interactions with other molecules. The corrosion inhibition 
efficiency calculated from theoretical calculation using conventional $\left(I E_{\text {the }} \%\right)$ and modified $\left(I E_{\mathrm{the}}^{*} \%\right)$ QSAR models along with their relative errors are summarized in Table 4.

It can be clearly seen that at different concentrations of all twelve Schiff bases, the relative errors obtained from MQSAR are less than relative errors obtained from QSAR model.

Table 3. Quantum chemical parameters of compounds SB1-SB12 using B3LYP/6-31G** calculations.

\begin{tabular}{cccc}
\hline SB & $\boldsymbol{E}_{\text {номо }}(\mathbf{e v})$ & $\boldsymbol{E}_{\text {LUMo }}(\mathbf{e v})$ & $\boldsymbol{\mu}(\mathbf{D})$ \\
\hline SB1 & -0.220 & -0.051 & 5.232 \\
SB2 & -0.203 & -0.057 & 8.171 \\
SB3 & -0.221 & -0.067 & 4.680 \\
SB4 & -0.226 & -0.065 & 3.588 \\
SB5 & -0.211 & -0.056 & 4.340 \\
SB6 & -0.179 & -0.037 & 5.749 \\
SB7 & -0.186 & -0.039 & 5.485 \\
SB8 & -0.177 & -0.049 & 3.396 \\
SB9 & -0.255 & -0.088 & 6.627 \\
SB10 & -0.229 & -0.076 & 3.479 \\
SB11 & -0.252 & -0.096 & 6.477 \\
SB12 & -0.250 & -0.097 & 4.990 \\
\hline
\end{tabular}

Table 4. The theoretical inhibition efficiency based on conventional $\left(I E_{\text {the }} \%\right)$ and $\operatorname{modified}\left(I E_{\text {the }}^{*} \%\right)$ QSAR models, where $E_{\mathrm{r}}$ and $E_{\mathrm{r}}^{*}$ are relative errors related to conventional and modified models, respectively, for the structures of the SB1-SB12 in different concentrations.

\begin{tabular}{ccccccc}
\hline SB & $\boldsymbol{C}(\mathbf{m M})$ & $\boldsymbol{I} \boldsymbol{E}_{\exp } \boldsymbol{*}$ & $\boldsymbol{I} \boldsymbol{E}_{\text {the }} \boldsymbol{*}$ & $\boldsymbol{I}_{\text {the }}^{*} \boldsymbol{\%}$ & $\boldsymbol{E}_{\mathbf{r}}$ & $\boldsymbol{E}_{\mathbf{r}}^{*}$ \\
\hline \multirow{2}{*}{ SB1 } & 0.10 & 89.77 & 89.41 & 89.95 & 0.401 & 0.200 \\
& 0.40 & 95.53 & 97.12 & 94.71 & 1.664 & 0.858 \\
\hline \multirow{2}{*}{ SB2 } & 0.05 & 97.60 & 91.80 & 91.88 & 5.943 & 5.861 \\
\hline \multirow{2}{*}{ SB3 } & 0.08 & 91.97 & 93.80 & 93.02 & 1.990 & 1.142 \\
& 0.32 & 97.33 & 98.37 & 96.39 & 1.068 & 0.966 \\
\hline \multirow{2}{*}{ SB4 } & 0.10 & 91.13 & 93.71 & 92.84 & 2.831 & 1.876 \\
& 0.21 & 94.87 & 96.90 & 94.95 & 2.140 & 0.084 \\
\hline \multirow{2}{*}{ SB5 } & 0.14 & 93.57 & 96.53 & 94.88 & 3.163 & 1.400 \\
& 0.21 & 95.30 & 97.66 & 95.78 & 2.476 & 0.504 \\
\hline
\end{tabular}




\begin{tabular}{ccccccc}
\hline \multirow{2}{*}{ SB } & $\boldsymbol{C}(\mathbf{m M})$ & $\boldsymbol{I} \boldsymbol{E}_{\exp } \boldsymbol{\%}$ & $\boldsymbol{I} \boldsymbol{E}_{\text {the }} \boldsymbol{\%}$ & $\boldsymbol{I}_{\text {the }}^{*} \boldsymbol{\%}$ & $\boldsymbol{E}_{\mathbf{r}}$ & $\boldsymbol{E}_{\mathbf{r}}^{*}$ \\
\hline \multirow{2}{*}{ SB6 } & 0.03 & 92.90 & 90.27 & 92.24 & 2.831 & 0.710 \\
& 0.10 & 95.90 & 96.87 & 95.60 & 1.011 & 0.313 \\
\hline \multirow{2}{*}{ SB7 } & 0.10 & 91.50 & 96.43 & 95.07 & 5.388 & 3.902 \\
& 1.00 & 97.43 & 99.63 & 98.39 & 2.258 & 0.985 \\
\hline SB8 & 0.10 & 93.50 & 97.74 & 96.69 & 4.535 & 3.412 \\
\hline SB9 & 1.20 & 93.40 & 97.79 & 95.05 & 4.700 & 1.766 \\
\hline SB10 & 0.03 & 90.27 & 85.68 & 89.63 & 5.085 & 0.709 \\
\hline \multirow{2}{*}{ SB11 } & 0.20 & 92.23 & 95.85 & 93.39 & 3.925 & 1.258 \\
& 1.20 & 95.23 & 99.28 & 97.19 & 4.253 & 2.058 \\
\hline \multirow{2}{*}{ SB12 } & 0.20 & 94.37 & 96.88 & 94.71 & 2.660 & 0.360 \\
& 1.20 & 96.60 & 99.46 & 97.77 & 2.961 & 1.211 \\
\hline
\end{tabular}

In order to make a statistical comparison between the relative errors obtained from QSAR and MQSAR models, the conical diagrams are depicted in Figure 5. Where, the relative error respect to MQSAR and QSAR models are identified by red and blue cones, respectively. Moreover, the standard deviation parameters for MQSAR and QSAR were calculated around 2.0 and 3.0 respectively, which confirm the superiority of MQSAR model.

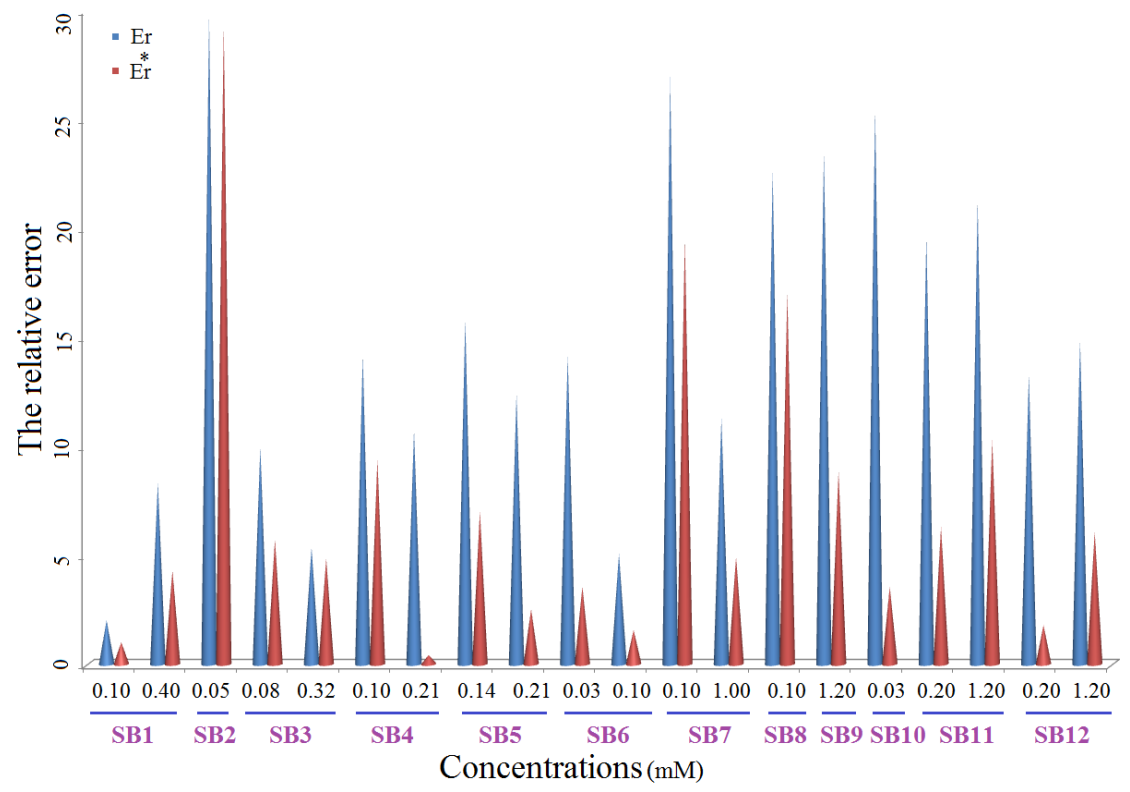

Figure 5. A comparison between relative errors for three Schiff bases SB1-SB12 in different concentrations, where the blue and red cones are related to QSAR and MQSAR models, respectively. 


\section{Conclusion}

In conclusion, we found some new Schiff bases having high corrosion inhibition efficiencies especially at high concentrations. Despite using the conventional nonlinear QSAR model for determination of the inhibition efficiency, there is still a significant gap between theoretical and experimental results in some cases. In addition to the new structures obtained in this study, we also evaluated our proposed model by different structures such as triazole and oxadiazole and thiadiazole derivatives at different concentrations. The results obtained from our proposed MQSAR model confirmed the superiority of this model compared to QSAR. However, further evaluations by a wide range of structures are needed in order to confirm the comprehensiveness of our proposed model. While using a favorable QSAR relationship for some structures would be very challenging, due to the divergence of the results from the experimental values, experimental verification can now be undertaken using the simulation results of this work.

\section{Conflicts of interest}

There are no conflicts to declare.

\section{References}

1. X. Zhang, K. Jiao, J. Zhang and Z. Guo, A review on low carbon emissions projects of steel industry in the World, J. Cleaner Prod., 2021, 306, 127259. doi: 10.1016/j.jclepro.2021.127259

2. O. Sanni, O.S.I. Fayomi and A.P.I. Popoola, Eco-friendly inhibitors for corrosion protection of stainless steel: an overview, J. Phys.: Conf. Ser., 2019, 1378, no. 4, 042047. doi: $10.1088 / 1742-6596 / 1378 / 4 / 042047$

3. U. Ekpe, U. Ibok, B. Ita, O. Offiong and E. Ebenso, Inhibitory action of methyl and phenyl thiosemicarbazone derivatives on the corrosion of mild steel in hydrochloric acid, Mater. Chem. Phys., 1995, 40, no. 2, 87-93. doi: 10.1016/0254-0584(94)01464-R

4. A.A.H. Kadhum, A.B. Mohamad, L.A. Hammed, A.A. Al-Amiery, N.H. San and A.Y. Musa, Inhibition of mild steel corrosion in hydrochloric acid solution by new coumarin, Materials, 2014, 7, no. 6, 4335-4348. doi: 10.3390/ma7064335

5. M. Quraishi and R. Sardar, Corrosion inhibition of mild steel in acid solutions by some aromatic oxadiazoles, Mater. Chem. Phys., 2003, 78, no. 2, 425-431. doi: 10.1016/S0254-0584(02)00299-7

6. K. Athul, P. Thilagavathy and D. Nalini, Inhibitory action of Macaranga peltata leaves extract on the corrosion of mild steel in $0.5 \mathrm{M}$ sulphuric Acid-Quantum chemical approach, Mater. Today: Proc., 2021, 47, 858-862. doi: 10.1016/j.matpr.2021.03.446

7. I.-M. Chung, R. Malathy, R. Priyadharshini, V. Hemapriya, S.-H. Kim and M. Prabakaran, Inhibition of mild steel corrosion using Magnolia kobus extract in sulphuric acid medium, Mater. Today Commun., 2020, 25, 101687. doi: $\underline{10.1016 / j . m t c o m m .2020 .101687}$ 
8. M.A. Khaled, M.A. Ismail, A.A. El-Hossiany and A.E.A.S. Fouda, Novel pyrimidinebichalcophene derivatives as corrosion inhibitors for copper in $1 \mathrm{M}$ nitric acid solution, RSC Adv., 2021, 11, 25314-25333. doi: 10.1039/D1RA03603C

9. A. Fawzy, T.A. Farghaly, A.A.Al Bahir, A.M. Hameed, A. Alharbi and Y.A. El-Ossaily, Investigation of three synthesized propane bis-oxoindoline derivatives as inhibitors for the corrosion of mild steel in sulfuric acid solutions, J. Mol. Struct., 2021, 1223, 129318. doi: 10.1016/j.molstruc.2020.129318

10. H. Lgaz, R. Salghi, K.S. Bhat, A. Chaouiki and S. Jodeh, Correlated experimental and theoretical study on inhibition behavior of novel quinoline derivatives for the corrosion of mild steel in hydrochloric acid solution, J. Mol. Liq., 2017, 244, 154-168. doi: 10.1016/j.molliq.2017.08.121

11. B. Ita and O. Offiong, The study of the inhibitory properties of benzoin, benzil, benzoin(4-phenylthiosemicarbazone) and benzil-(4-phenylthiosemicarbazone) on the corrosion of mild steel in hydrochloric acid, Mater. Chem. Phys., 2001, 70, no. 3, 330-335. doi: 10.1016/S0254-0584(00)00476-4

12. M.N. Desai and M.B. Desai, Carbonyl compounds as corrosion inhibitors for mild steel in $\mathrm{HCl}$ solutions, Corros. Sci., 1984, 24, no. 8, 649-660. doi: 10.1016/0010938X(84)90056-8

13. G. Makovej, V. Ushakov, V. Bagin and A. Tolmachev, Electrochemical corrosion behaviour of iron in hydrochloric solutions inhibited by $\gamma$-irradiated 2aminobenzothiazole, Ukr. Khim. Zh., 1988, 54, no. 5, 505-508.

14. P.B. Raja, M. Ismail, S. Ghoreishiamiri, J. Mirza, M.C. Ismail, S. Kakooei and A.A. Rahim, Reviews on corrosion inhibitors: a short view, Chem. Eng. Commun., 2016, 203, no. 9, 1145-1156. doi: 10.1080/00986445.2016.1172485

15. H. Gilson, B.H. Honig, A. Croteau, G. Zarrilli and K. Nakanishi, Analysis of the factors that influence the $\mathrm{C}=\mathrm{N}$ stretching frequency of polyene Schiff bases. Implications for bacteriorhodopsin and rhodopsin, Biophys. J., 1988, 53, no. 2, 261-269. doi: 10.1016/S0006-3495(88)83087-X

16. T. Baasov, N. Friedman and M. Sheves, Factors affecting the $C: N$ stretching in protonated retinal Schiff base: a model study for bacteriorhodopsin and visual pigments, Biochemistry, 1987, 26, no. 11, 3210-3217. doi: 10.1021/bi00385a041

17. H.P. Ebrahimi, J.S. Hadi, Z.A. Abdulnabi and Z. Bolandnazar, Spectroscopic, thermal analysis and DFT computational studies of salen-type Schiff base complexes, Spectrochim. Acta, Part A, 2014, 117, 485-492. doi: 10.1016/j.saa.2013.08.044

18. J. Ortega-Castro, M. Adrover, J. Frau, A. Salvà, J. Donoso and F. Muñoz, DFT studies on Schiff base formation of vitamin B6 analogues. Reaction between a pyridoxamineanalogue and carbonyl compounds, J. Phys. Chem. A, 2010, 114, no. 13, 4634-4640. doi: $10.1021 / \mathrm{jp} 909156 \mathrm{~m}$

19. N.O. Eddy and B.I. Ita, QSAR, DFT and quantum chemical studies on the inhibition potentials of some carbozones for the corrosion of mild steel in $\mathrm{HCl}, \mathrm{J}$. Mol. Model., 2011, 17, no. 2, 359-376. doi: 10.1007/s00894-010-0731-7 
20. M. Hadizadeh, L. Yang, G. Fang, Z. Qiu and Z. Li, Mobility and Solvation Structure of Hydroxyl Radical in a Water Nanodroplet: A Born-Oppenheimer Molecular Dynamics Study, Phys. Chem. Chem. Phys., 2021, 23, 14628-14635. doi: 10.1039/D1CP01830B

21. M. Hadizadeh and M. Hamadanian, Adsorption of toxic gases by an open nanocone coupled with an iron atom, Bulg. Chem. Commun., 2014, 46, no. 3, 576-579.

22. M. Hadizadeh, DFT study of carbon monoxide adsorption on zinc oxide nanocone, Bulg. Chem. Commun., 2016, 48, no. 1, 105-108.

23. S. Zhang, W. Lei, M. Xia and F. Wang, QSAR study on N-containing corrosion inhibitors: quantum chemical approach assisted by topological index, J. Mol. Struct.: THEOCHEM, 2005, 732, no. 1-3, 173-182. doi: 10.1016/j.theochem.2005.02.091

24. W.P. Singh and J. Bockris, Toxicity issues of organic corrosion inhibitors: applications of QSAR model, Corrosion, 96, 1996.

25. K. Khaled, Modeling corrosion inhibition of iron in acid medium by genetic function approximation method: A QSAR model, Corros. Sci., 2011, 53, no. 11, 3457-3465. doi: $\underline{10.1016 / j . c o r s c i .2011 .01 .035}$

26. M. Shabani-Nooshabadi, M. Behpour, F.S. Razavi, M. Hamadanian and V. Nejadshafiee, Study of N-benzylidene derivatives synthesized as corrosion inhibitors for copper in $\mathrm{HCl}$ solution, $R S C A d v$., 2015, 5, no. 30, 23357-23366. doi: 10.1039/C5RA00561B

27. M. Behpour, A. Attaran, S. Ghoreishi, N. Mohammadi, M. Hamadanian, M. Salavati-Niasari and M. Abbasi, Investigation of adsorption and inhibitive properties of some diamine compounds on mild steel corrosion in hydrochloric acid solution, Chem. Eng. Commun., 2014, 201, no. 8, 1077-1095. doi: 10.1080/00986445.2013.803074

28. M. Behpour, S. Ghoreishi, F. Vatani, N. Mohammadi and M. Ghasemzadeh, The inhibiting effect of some new derivatives of pyrimidine-2-thione on the corrosion of stainless steel 304 in sulfuric acid media, J. Mech. Eng. Tech., 2014, 2, 1-11.

29. M. Behpour, S. Ghoreishi, N. Soltani, M. Salavati-Niasari, M. Hamadanian and A. Gandomi, Electrochemical and theoretical investigation on the corrosion inhibition of mild steel by thiosalicylaldehyde derivatives in hydrochloric acid solution, Corros. Sci., 2008, 50, no. 8, 2172-2181. doi: 10.1016/j.corsci.2008.06.020

30. I. Obot, N. Obi-Egbedi and S. Umoren, Antifungal drugs as corrosion inhibitors for aluminium in $0.1 \mathrm{M} \mathrm{HCl}$, Corros. Sci., 2009, 51, no. 8, 1868-1875. doi: 10.1016/j.corsci.2009.05.017

31. M. Frisch, G. Trucks, H.B. Schlegel, G.E. Scuseria, M.A. Robb, J.R. Cheeseman, G. Scalmani, V. Barone, B. Mennucci and G. Petersson, Gaussian 09, Revision d. 01, Gaussian, Inc., Wallingford CT, 2009, 201.

32. A.D. Becke, Density-functional exchange-energy approximation with correct asymptotic behavior, Phys. Rev. A, 1988, 38, no. 6, 3098. doi: 10.1103/PhysRevA.38.3098

33. C. Lee, W. Yang and R.G. Parr, Development of the Colle-Salvetti correlation-energy formula into a functional of the electron density, Phys. Rev. B, 1988, 37, no. 2, 785. doi: $\underline{10.1103 / \text { PhysRevB.37.785 }}$ 
34. O. Tapia, Solvent effect theories: Quantum and classical formalisms and their applications in chemistry and biochemistry, J. Math. Chem., 1992, 10, no. 1, 139-181. doi: $10.1007 / \mathrm{BF} 01169173$

35. B.I.A. Simkin and I.I. Sheikkhet, Quantum chemical and statistical theory of solutions: $a$ computational approach, Ellis Horwood, London, 1995.

36. J. Tomasi and M. Persico, Molecular interactions in solution: an overview of methods based on continuous distributions of the solvent, Chem. Rev., 1994, 94, no. 7, 2027-2094. doi: $10.1021 / \mathrm{cr} 00031 \mathrm{a} 013$

37. M. Cossi, V. Barone, R. Cammi and J. Tomasi, Ab initio study of solvated molecules: a new implementation of the polarizable continuum model, Chem. Phys. Lett., 1996, 255, no. 4-6, 327-335. doi: 10.1016/0009-2614(96)00349-1

38. V. Barone, M. Cossi and J. Tomasi, Geometry optimization of molecular structures in solution by the polarizable continuum model, J. Comput. Chem., 1998, 19, no. 4, 404-417.

39. H. El Ashry, A. El Nemr, S. Essawy and S. Ragab, Corrosion inhibitors part IV: Quantum chemical studies on the corrosion inhibition of steel in acidic medium by some aniline derivatives, J. Phys. Chem., 2006, 1, 41-55.

40. I. Lukovits, A. Shaban and E. Kálmán, Corrosion inhibitors: quantitative structureactivity relationships, Russ. J. Electrochem., 2003, 39, no. 2, 177-181. doi: 10.1023/A:1022313126231

41. M. Lebrini, F. Bentiss, H. Vezin and M. Lagrenée, Inhibiting effects of some oxadiazole derivatives on the corrosion of mild steel in perchloric acid solution, Appl. Surf. Sci., 2005, 252, no. 4, 950-958. doi: 10.1016/j.apsusc.2005.01.160

42. M. Lebrini, M. Lagrenee, H. Vezin, L. Gengembre and F. Bentiss, Electrochemical and quantum chemical studies of new thiadiazole derivatives adsorption on mild steel in normal hydrochloric acid medium, Corros. Sci., 2005, 47, no. 2, 485-505. doi: 10.1016/j.corsci.2004.06.001

43. F. Bentiss, M. Traisnel, H. Vezin, H. Hildebrand and M. Lagrenee, 2,5-Bis(4dimethylaminophenyl)-1,3,4-oxadiazole and 2,5-bis(4-dimethylaminophenyl)-1,3,4thiadiazole as corrosion inhibitors for mild steel in acidic media, Corros. Sci., 2004, 46, no. 11, 2781-2792. doi: 10.1016/j.corsci.2004.04.001

44. F. Bentiss, M. Lebrini, H. Vezin and M. Lagrenée, Experimental and theoretical study of 3-pyridyl-substituted 1,2,4-thiadiazole and 1,3,4-thiadiazole as corrosion inhibitors of mild steel in acidic media, Mater. Chem. Phys., 2004, 87, no. 1, 18-23. doi: 10.1016/j.matchemphys.2004.05.040

45. F. Bentiss, M. Traisnel, H. Vezin and M. Lagrenée, Linear resistance model of the inhibition mechanism of steel in $\mathrm{HCl}$ by triazole and oxadiazole derivatives: structureactivity correlations, Corros. Sci., 2003, 45, no. 2, 371-380. doi: 10.1016/S0010938X(02)00102-6 
46. F. Bentiss, M. Traisnel, N. Chaibi, B. Mernari, H. Vezin and M. Lagrenée, 2,5-Bis(nmethoxyphenyl)-1,3,4-oxadiazoles used as corrosion inhibitors in acidic media: correlation between inhibition efficiency and chemical structure, Corros. Sci., 2002, 44, no. 10, 2271-2289. doi: 10.1016/S0010-938X(02)00037-9

47. M. Lagrenee, B. Mernari, M. Bouanis, M. Traisnel and F. Bentiss, Study of the mechanism and inhibiting efficiency of 3,5-bis(4-methylthiophenyl)-4H-1,2,4-triazole on mild steel corrosion in acidic media, Corros. Sci., 2002, 44, no. 3, 573-588. doi: 10.1016/S0010-938X(01)00075-0

48. M. Lagrenee, B. Mernari, N. Chaibi, M. Traisnel, H. Vezin and F. Bentiss, Investigation of the inhibitive effect of substituted oxadiazoles on the corrosion of mild steel in $\mathrm{HCl}$ medium, Corros. Sci., 2001, 43, no. 5, 951-962. doi: 10.1016/S0010-938X(00)00076-7

49. F. Bentiss, M. Traisnel and M. Lagrenee, The substituted 1,3,4-oxadiazoles: a new class of corrosion inhibitors of mild steel in acidic media, Corros. Sci., 2000, 42, no. 1, 127-146. doi: 10.1016/S0010-938X(99)00049-9

50. F. Bentiss, M. Traisnel, L. Gengembre and M. Lagrenée, Inhibition of acidic corrosion of mild steel by 3,5-diphenyl-4H-1,2,4-triazole, Appl. Surf. Sci., 2000, 161, no. 1-2, 194-202. doi: 10.1016/S0169-4332(00)00287-7 